\title{
Enhancing Syndromic Surveillance with Procedure Data: A 2017-8 Influenza Case Study
}

\section{Andrew Walsh}

Health Monitoring, Pittsburgh, Pennsylvania, United States

Objective

To identify additional data elements in existing syndromic surveillance message feeds that can provide additional insight into public health concerns such as the influenza season.

\section{Introduction}

Syndromic surveillance achieves timeliness by collecting prediagnostic data, such as emergency department chief complaints, from the start of healthcare interactions. The tradeoff is less precision than from diagnosis data, which takes longer to generate. As the use and sophistication of electronic health information systems increases, additional data that provide an intermediate balance of timeliness and precision are becoming available.

Information about the procedures and treatments ordered for a patient can indicate what diagnoses are being considered. Procedure records can also be used to track the use of preventive measures such as vaccines that are also relevant to public health surveillance but not readily captured by typical syndromic data elements.

Some procedures such as laboratory tests also provide results which can provide additional specificity about which diagnoses will be considered. If procedure and treatment orders and test results are included in existing syndromic surveillance feeds, additional specificity can be achieved with timeliness comparable to prediagnostic assessments.

\section{Methods}

HL7 messages were collected for syndromic surveillance using EpiCenter software. They were retroactively scanned for PR1 procedure segments; procedure codes and descriptions were extracted when available. Influenza-related procedures were identified and classified as either a test for the virus or an administration of a vaccine. Classification was based on the procedure code when a standard code set was used and could be identified, otherwise it was based on the text description of the procedure.

Messages were also scanned for the presence of 'influenza' in text fields. Influenza test results were identified first by selecting messages with 'influenza' in an OBX segment and then further refining based on the test code and description.

\section{Results}

A total of 443,074,748 messages from 2,577 healthcare facilities received between July 1, 2017 and August 31, 2018 were scanned for procedure information. Procedure codes were present in 39,142,670 messages from 287 facilities. The most common procedures included blood glucose measurements and other diabetes maintenance activities, incentive spirometry, blood count and metabolic panels, safety observation, and vital signs.

Of those, 995,754 messages from 142 facilities contained influenza-related procedure codes for 106,610 visits. 14,672 visits from 62 facilities had one of 48 vaccine procedure codes, and 91, 948 visits from 127 facilities had one of 66 test codes. Time series of both types of procedures showed a seasonal trend consistent with the influenza season. Figure 1 shows the daily counts of influenza test orders and vaccine administrations. Figure 2 breaks out the test orders by test type (antibody assay, antigen assay, PCR, or unspecified).

Seven facilities sent a total of 58,182 messages containing influenza test results. These included both positive and negative results. These results distinguished between influenza A and influenza B. Figure 3 shows the daily counts of both positive and negative results by virus type; this also follows the expected seasonal pattern.

SDS Annual Conference Proceedings 2019. This is an Open Access article distributed under the terms of the Creative Commons AttributionNoncommercial 4.0 Unported License (http://creativecommons.org/licenses/by-nc/3.0/), permitting all non-commercial use, distribution, and reproduction in any medium, provided the original work is properly cited. 


\section{Conclusions}

Since procedure information was not specifically requested from healthcare facilities, the overall representation of procedure data elements was low. These initial results indicate that such data would be useful both as a supplement to syndromic surveillance activities and as a new data source for other surveillance activities such as vaccine uptake tracking. Given the frequency of procedures and treatments for chronic diseases such as diabetes and heart disease, these data may be relevant for understanding the prevalence of those conditions as well. Tests and treatments relevant to other public health concerns like opioid use disorder were also present, suggesting a wide range of potential applications.

It is also possible to obtain and extract influenza test results from these syndromic surveillance messages. Both positive and negative results were present, providing information not just on the number of positive cases but also the rate of testing and rate of positive results. The pattern of testing and results also indicates that at least some facilities test for influenza throughout the season, contrary to some conventional wisdom about testing patterns.

\section{Acknowledgement}

Health Monitoring would like to thank our customers for financial support of this work.

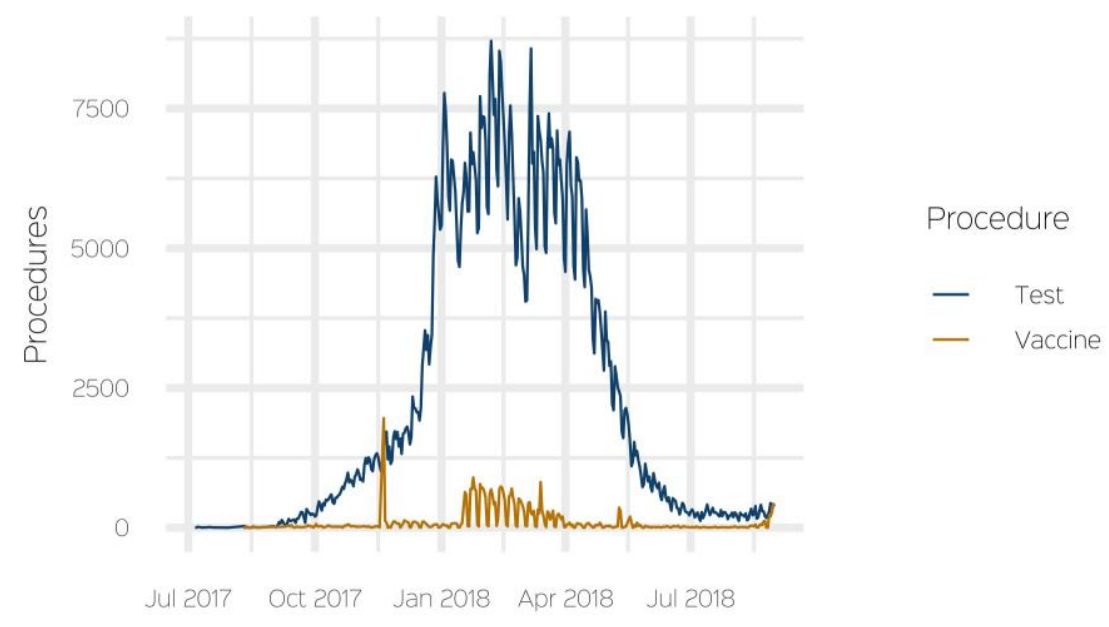

Figure 1: Time Series of Influenza-Related Procedure Orders by Procedure Type

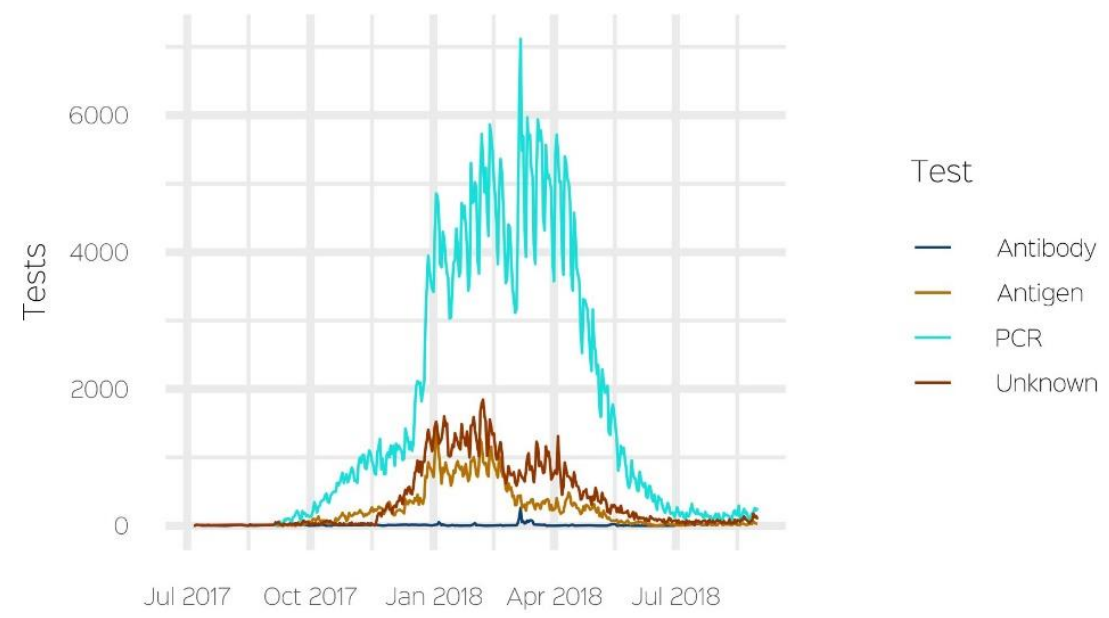

Figure 2: Time Series of Influenza-Related Test Orders by Test Type 


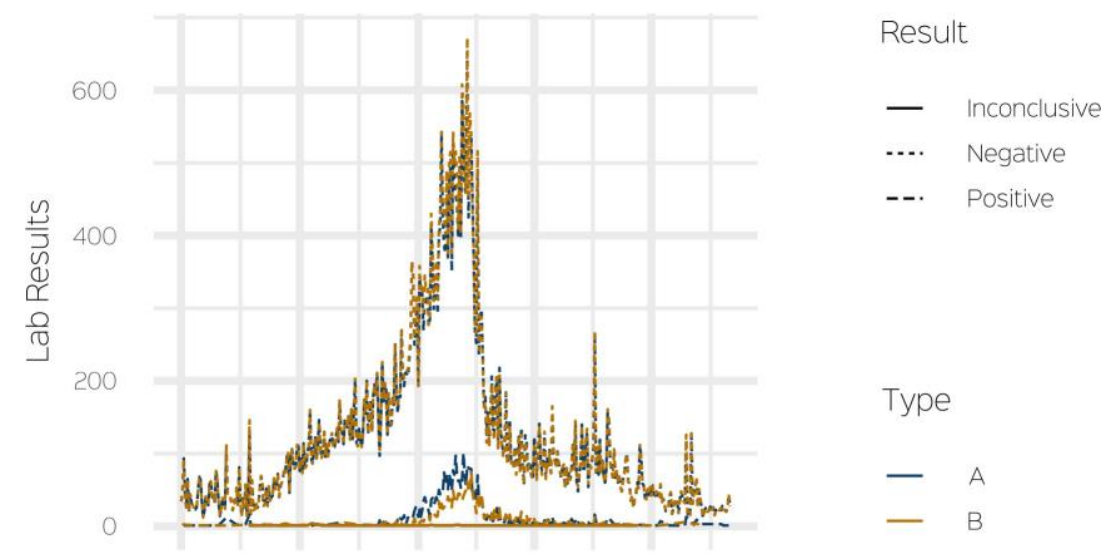

Jul2017 Oct 2017 Jan 2018 Apr 2018 Jul 2018

Figure 3: Time Series of Influenza Test Results by Result and Influenza Type 